The Journal of Engineering and Exact Sciences - jCEC, Vol. 06 N. 03 (2020)

journal homepage: https://periodicos.ufv.br/ojs/jcec

doi: 10.18540/jcecvl6iss3pp0421-0428

OPEN ACCESS - ISSN: 2527-1075

\title{
ON APPROXIMATION OF SUM OF CONVERGENT SERIES
}

D.R. Paudyal ${ }^{1, *}$, Lakshmi Narayan Mishra ${ }^{2}$

${ }^{1}$ Tribhuvan University, Department of Mathematics-Birendra Multiple Campus, Chitwan, Kathmandu Bagmati Pradesh, Nepal.

ORCID: https://orcid.org/0000-0002-7128-3242

${ }^{2}$ Vellore Institute of Technology University, Department of Mathematics, School of Advance Sciences, Vellore 632 014, Tamil Nadu, India. Orcid.org/

ORCID: https://orcid.org/0000-0001-7774-7290

*Corresponding author: Tribhuvan University, Department of Mathematics-Birendra Multiple Campus, Chitwan, Kathmandu, Bagmati Pradesh, Nepal, Mob.0977-9845080164,E-mail: dayaram_paudyal@yahoo.com

\section{A R T I C LE IN F O}

Article history:

Received 2020-06-20

Accepted 2020-09-04

Available online 2020-09-04

keywords

Bernoulli numbers

Convergent series

Infinite series

Partial sum
A B S T R A C T

The paper has given a clear opinion on the progress of environmental protection and sustainability in the Nigerian context. The environmental regulations scenario in the country is marred by malpractices and corruption more stringent policy enforcement will help in the achievement of environmental protection. This paper deals with a specialized method of approximating the sum of an infinite series containing positive terms which are monotonically decreasing. The analysis has been done by taking some references done by the great mathematician Leonhard Euler with some special examples. Consequently, we have established a relation to estimate the sum of convergent infinite series. 


\section{INTRODUCTION}

In the $17^{\text {th }}$ and $18^{\text {th }}$ century many contributions have been done in the direction of infinite series. The major contributions is due to the great mathematician Leonhard Euler (1707-1783) [Linda Becerra and et al.,2008]. Out of his major works finding Euler numbers and zeta functions are of very genius. Moreover, he attempted to find the sum of different types of infinite series. The classical concept of convergent of infinite series was put on reasonable foundation with the contribution of Cauchy monumental work and Abel's research on the binomial series. A precise definition for the concept of a convergent series was introduced at the beginning of the $19^{\text {th }}$ century by Augstin Cauchy (1789-1857) and Niles Henrik Abel (1802-1829). Depending upon Euler's study it has already been established that a standard method of finding the sum of infinite series is to find the partial sum and to evaluate its limit. The concept of infinite sum of positive terms is mysterious and very interesting. Pietro Mengoli (1626-1686) used infinite series to good effect in Novae quadraturaearithmeticae, sue de additionefractionum published in Bologna in 1650, developing ideas which had first been investigated by Cataldi (1548-1626). $\mathrm{He}$ begins with the summation of geometric series and he shows that the harmonic series does not converge. He became the first person to prove that it was possible for a series whose terms tend to zero to be made larger than any given number. If the series is very slowly convergent and it is difficult to find the partial sum that converges to a limit then we find partial sum $S_{n}$ by direct addition of $\mathrm{n}$ terms or by any existing methods in which we add the remainder $\mathrm{R}_{\mathrm{n}}$ for the remaining terms. Even then to minimize the errors in our estimated sum, the direct addition of many more terms is needed which is also a tedious task. That is why many mathematicians have attempted to transform the series in different forms. Euler's, Kummer's and Markoff's transformations are ingenious method in this field [Becerra et al., 2008; Stanattis,2009; Pfaff, 2007]. Many of the infinite series were already studied by many earlier mathematicians but Euler established many ideas of finding the sum of infinite series of positive terms by publishing some papers in 1735 and 1740 [Gautschi, 2008]. Euler transformed the infinite series

$\sum_{k=1}^{\infty} \frac{1}{k^{2}}=\frac{1}{1^{2}}+\frac{1}{2^{2}}+\frac{1}{3^{2}}+\frac{1}{4^{2}}+\ldots$

into

$\sum_{k=1}^{\infty} \frac{1}{k^{2} \times 2^{k-1}}+(\ln 2)^{2}$

For it he used an improper integral given by

$\mathrm{I}=\int_{0}^{1 / 2}-\frac{\ln (1-t)}{t} d t \quad$ and estimated the infinite sum of $\sum_{k=1}^{\infty} \frac{1}{k^{2}}=1.644934$ by taking only14 terms. He finally declared that its actual sum is $\frac{\pi^{2}}{6}$ [Dunham,1999]. In a paper of 1740 Euler obtained one of his finest triumphs given by
$S_{2 n}=\sum_{v=1}^{\infty} \frac{1}{v^{2 n}}=(-1)^{n} \frac{2 n}{2 \times(2 n) !} B_{2 n} \quad$ [Kline, 1983].

Here $B_{2 n}$ are Bernouli numbers given as

$$
B_{2}=\frac{1}{6}, B_{4}=-\frac{1}{30}, B_{6}=\frac{1}{42}, B_{8}=-\frac{1}{30}, B_{10}=\frac{5}{66}, \ldots
$$

Our concern, now, is to find the sum of infinite series of positive terms with reference to Euler's finding.

\section{PRELIMINARIES}

Definition: Let $\sum_{n=0}^{\infty} a_{n}$ be an infinite series having $\mathrm{n}^{\text {th }}$ partial sum $\mathrm{S}_{\mathrm{n}}=\sum_{k=0}^{n} a_{k}$. Such infinite series is said to be convergent to the sum S if $\lim _{n \rightarrow \infty} S_{n}$ exists where $\left\{\mathrm{S}_{\mathrm{n}}\right\}$ is the sequence of partial sums. If the sequence of partial sums has no limit then the series is said to be divergent [Knoop,1990].

\section{Basel Problem- Euler's an Attempt}

The Basel problem is to find the sum of series $\sum_{k=1}^{\infty} \frac{1}{k^{2}}=\frac{1}{1^{2}}+\frac{1}{2^{2}}+\frac{1}{3^{2}}+\frac{1}{4^{2}}+\ldots$

Of course, one can use the calculator to estimate the sum. Adding up 10 terms gives 1.55 but it doesn't tell us much about the whole sum. If we add up to 200 terms then the correct two decimal approximations is 1.64. In other hands, the harmonic series which has an infinite sum gives total sum less than 6 though we take 200 terms. For these reasons we can say that the direct calculation is not so fruitful. So alternative methods are needed to make accurate estimates of the sum other than direct calculations. Euler worked on such infinite series. By adding the first fewer and 100 terms he claimed that the sum of the series $\sum_{k=1}^{\infty} \frac{1}{k^{2}}$ is nearest about 1.54977 (10 terms) and is nearest about 1.63498 (100 terms). By these sums it is concluded that the sum of these series converges very slowly. In 1731 Euler published a paper in which he found a way to improve dramatically such numerical approximations. This discovery is assumed as a remarkable work in its vision and fearless in its manipulation. The symbols he used were truly ingenious [Erdos et al., 1983]. In various ways Euler attempted the Bassel problem. One of the techniques he used is discussed as below. He used the improper integral

$$
I=\int_{0}^{1 / 2}-\frac{\ln (1-t)}{t} d t \quad ; t \in\left(0, \frac{1}{2}\right]
$$




$$
=\int_{0}^{1 / 2}-\frac{\left(-t-t^{2} / 2-t^{3} / 3-t^{4} / 4-\ldots \ldots\right)}{t} d t
$$

$$
=\int_{0}^{1 / 2}\left(1+t / 2+t^{2} / 3+t^{3} / 4+t^{4} / 5+\ldots .\right) d t=\frac{1}{2}+\frac{1 / 2^{2}}{4}+\frac{1 / 2^{3}}{9}+\frac{1 / 2^{4}}{16}+\ldots
$$

Again, he attempted it by putting

$$
1-\mathrm{t}=\mathrm{z} \text { in }(2.2) ; \quad z \in\left[\frac{1}{2}, 1\right)
$$

so that $I=\int_{1}^{1 / 2} \frac{\ln z}{1-z} d z=\int_{1}^{1 / 2} \ln z(1-z)^{-1} d z=\int_{1}^{1 / 2}\left(1+z+z^{2}+z^{3}+\ldots\right) \ln z d z$

It is also true that

$$
\int_{1}^{1 / 2} z^{n} \ln z d z=\left[\frac{z^{n+1}}{n+1} \ln z-\frac{z^{n+1}}{(n+1)^{2}}\right]_{1}^{1 / 2} \quad \text { (By integration by parts) }
$$

By using (2.5) in (2.4) it is obtained that

$$
\begin{aligned}
& I=\left[(z \ln z-z)+\left(\frac{z^{2}}{2} \ln z-\frac{z^{2}}{4}\right)+\left(\frac{z^{3}}{3} \ln z-\frac{z^{3}}{9}\right)+\ldots \ldots\right]_{1}^{\frac{1}{2}} \\
& =\left[\ln z\left(z+\frac{z^{2}}{2}+\frac{z^{3}}{3}+\frac{z^{4}}{4}+\frac{z^{5}}{5}+\ldots . .\right)-\left(z+\frac{z^{2}}{4}+\frac{z^{3}}{9}+\frac{z^{4}}{16}+\frac{z^{5}}{25}+\ldots .\right)\right]_{1}^{1 / 2} \\
& =\left[\ln z(-\ln (1-z))-\left(z+\frac{z^{2}}{4}+\frac{z^{3}}{9}+\frac{z^{4}}{16}+\frac{z^{5}}{25}+\ldots\right)\right]_{1}^{1 / 2} \\
& =-\left(\ln \frac{1}{2}\right)^{2}-\left(\frac{1}{2}+\frac{\frac{1}{2^{2}}}{4}+\frac{1}{\frac{2^{3}}{9}}+\frac{1}{16}+\frac{1}{2^{5}}+\ldots .+(\ln (1-))(\ln (0+))+\sum_{k=1}^{\infty} \frac{1}{k^{2}}\right. \\
& I=-(\ln 2)^{2}-\left(\frac{1}{2}+\frac{\frac{1}{2^{2}}}{4}+\frac{1}{2^{3}}+\frac{1}{9}+\frac{1}{16}+\frac{2^{5}}{25}+\ldots . .0+\sum_{k=1}^{\infty} \frac{1}{k^{2}}\right.
\end{aligned}
$$

Using (2.3), it is obtained that

$I=-(\ln 2)^{2}-I+0+\sum_{k=1}^{\infty} \frac{1}{k^{2}}$ 


$$
\begin{aligned}
\therefore \sum_{k=1}^{\infty} \frac{1}{k^{2}} & =2\left(\frac{1}{2}+\frac{\frac{1}{2^{2}}}{4}+\frac{\frac{1}{2^{3}}}{9}+\frac{\frac{1}{2^{4}}}{16}+\frac{\frac{1}{2^{5}}}{25}+\ldots\right)+(\ln 2)^{2} \\
& =\sum_{k=1}^{\infty} \frac{1}{k^{2} \times 2^{k-1}}+(\ln 2)^{2}
\end{aligned}
$$

In this way he transformed the problem in next form and deduced the value. Using only fourteen terms he obtained that $\sum_{k=1}^{\infty} \frac{1}{k^{2}}=1.644934$.This is far more accurate than summing a thousand terms of the original series. Four year later, in 1735, Euler finally succeeded to find exact sum of $\sum_{k=1}^{\infty} \frac{1}{k^{2}}$ and announced this discovery in 1735 at the age of 28 . His arguments were based on manipulations that were not justified at that time. Later, he was able to produce a truly rigorous proof that the sum of above series (2.1) is $\frac{\pi^{2}}{6}$ [Pfaff, 2007; Paudyal, 2014; David, 2012].

Similarly, Kummer's and Markoff's transformations [David, 2012; Stanattis, 2009] of (2.1) are respectively given by

$$
\begin{aligned}
& \sum_{n=1}^{\infty} \frac{1}{n^{2}}=\mathrm{S}_{\mathrm{p}}+\mathrm{P} ! \sum_{\mathrm{n}=1}^{\infty} \frac{1}{\mathrm{n}^{2}(n+1)(n+2)(n+3)(n+4) \cdots(n+p)} ; \mathrm{p}=1,2,3 \cdots \\
& \sum_{n=1}^{\infty} \frac{1}{n^{2}}=3 \sum_{\mathrm{n}=1}^{\infty} \frac{[(n-1) !]^{2}}{(2 n) !}
\end{aligned}
$$

From (2.10) we get the sum accurate to 6 decimal places taking 17 terms $(p=9)$ while the relation (2.11) gives same accuracy taking only 9 terms. So, it is seen that (2.11) is fast convergent than (2.10). But both these relations are seemed useful for particular cases. Again, some mathematicians studied about the upper and lower estimate method given by Euler as in the following theorem.

Theorem 2.2.1: If $S=\sum_{x=1}^{\infty} f(x)$ is convergent series of $\mathrm{f}(\mathrm{x})$ defined for $\mathrm{x} \geq 1$ and it is $(2 \mathrm{~m}-1)$ times continuously differentiable then the approximate sum of this series is given by

$$
\begin{aligned}
& S \cong \sum_{x=1}^{k} f(x)+\int_{k+1}^{\infty} f(x) d x+\frac{1}{2} f(k+1)-\frac{B_{2}}{2 !} f^{i}(k+1)-\frac{B_{4}}{4 !} f^{i i i}(k+1)-\frac{B_{6}}{6 !} f^{v}(k+1) \cdots-\frac{B_{2 m}}{(2 m) !} f^{2 m-1}(k+1) \\
&+\int_{k+1}^{\infty} P_{2 m+1} f^{2 m+1}(x) d x, \text { where } \\
& P_{2 m+1}(x)=(-1)^{m-1} \sum \frac{2 \sin 2 \pi n x}{(2 \pi n)^{2 m-1}} \text { and } \mathrm{B}_{2 m} \text { are Bernoulli's numbers. }
\end{aligned}
$$

In this theorem, O.E. Stanattis et al. [Nick, 2014; Stanattis, 2009] argued that for the remainder $\mathrm{R}_{\mathrm{k}}$ we can have

$$
\begin{aligned}
& \left|R_{k}-\int_{k+1}^{\infty} f(x) d x+\frac{1}{2} f(k+1)-\frac{B_{2}}{2 !} f^{i}(k+1)-\frac{B_{4}}{4 !} f^{i i i}(k+1)-\frac{B_{6}}{6 !} f^{v}(k+1) \cdots-\frac{B_{2 m}}{(2 m) !} f^{2 m-1}(k+1)\right| \\
& \leq \int_{k+1}^{\infty} P_{2 m-1}(x) f^{2 m+1}(x) d x=r_{k} \quad(\text { say })
\end{aligned}
$$


They also argued that the remainder $r_{k}$ becomes such that

$r_{k}<\frac{B_{2 m}}{2 \pi(2 m) !} \int_{k+1}^{\infty}\left|f^{2 m+1}(x)\right| d x$

Thus they restated the above theorem in the following form [Stantis, 2009; Collins, 1992].

Theorem 2.2.2: If $S=\sum_{x=1}^{\infty} f(x)$ is convergent series where $\mathrm{f}(\mathrm{x})$ is defined for $\mathrm{x} \geq 1$ and it is $(2 \mathrm{~m}+1)$ times continuously differentiable then the error $\quad r_{k}$ of the approximation $S \cong \sum_{x=1}^{k} f(x)+\int_{k+1}^{\infty} f(x) d x+\frac{1}{2} f(k+1)-\frac{B_{2}}{2 !} f^{i}(k+1)-\frac{B_{4}}{4 !} f^{i i i}(k+1)-\frac{B_{6}}{6 !} f^{v}(k+1) \cdots-\frac{B_{2 m}}{(2 m) !} f^{2 m-1}(k+1)$

is $\int_{k+1}^{\infty} P_{2 m+1}(x) f^{2 m+1}(x) d x<\frac{B_{2 m}}{2 \pi(2 m) !} \int_{k+1}^{\infty}\left|f^{2 m+1}(x)\right| d x$

This instrument is stronger in the sense that it approximates the series (2.1) with error less than $10^{-7}$ when $\mathrm{k}=4$ and $\mathrm{m}=4$. By increasing the value of $\mathrm{k}$ and $\mathrm{m}$ it gives the sum equal to $1.6449340668482323 \ldots$ whose error is less than $10^{-16}$. Ehnberg, in 1966 , has also studied about the shortcut method of approximation of infinite series of positive terms [Ehenberg, 1966]. Similarly. Mishra [Mishra, 2007; Mishra et al., 2012] studied about the approximation of different functions in Banach space and established many theorems of transformations.

Theorem (Integral Test) 2.3.1: Let $\left\{a_{n}\right\}$ be a sequence of positive terms. Suppose $a_{n}=f(n)$, where $f$ is continuous, positive, decreasing function of $\mathrm{x}$ for all $\mathrm{x} \geq \mathrm{N}$, a positive integer. Then the series $\sum_{n=N}^{\infty} a_{n}$ and the integral $\int_{N}^{\infty} f(x) d x$ both converge or both diverge [Nelson,2003].

\section{MAIN THEOREMS}

Theorem 3.1: Let $\sum_{k=0}^{\infty}(a+k d) r^{k}$, where $-1<\mathrm{r}<1, \mathrm{a} \geq 0$ be an infinite series of positive terms which is monotonically decreasing then it is convergent and converges approximately to

$\sum_{k=0}^{n}(a+k d) r^{k}+\frac{r^{\frac{2 n+1}{2}}}{\ln r}\left[-a-\frac{d(2 n+1)}{2}+\frac{d}{\ln r}\right]$, for $\mathrm{n} \geq 5$

Proof: Here we have $\sum_{k=0}^{\infty}(a+k d) r^{k}$ which is an infinite series. First, we prove it is convergent. Assume $\mathrm{f}(\mathrm{x})=(\mathrm{a}+\mathrm{x} \mathrm{d}) \mathrm{r}$ where $x=1,2,3 \ldots$ Obviously, this function is an exponential function and hence it is continuous on [0, $\infty$ ). Now, the improper integral that describes the partial sum of the given series is given by

$$
\int_{\mathrm{N}}^{\infty}(\mathrm{a}+\mathrm{xd}) \mathrm{r}^{\mathrm{x}} d x=\lim _{t \rightarrow \infty} \int_{\mathrm{N}}^{t}(\mathrm{a}+\mathrm{xd}) \mathrm{r}^{\mathrm{x}} d x=\lim _{t \rightarrow \infty}\left[a \int_{N}^{t} r^{x} d x+d \int_{N}^{t} x r^{x} d x\right]
$$




$$
\begin{aligned}
& =\lim _{t \rightarrow \infty}\left[\frac{a r^{x}}{\ln r}+\frac{d x r^{x}}{\ln r}-\frac{d r^{x}}{(\ln r)^{2}}\right]_{N}^{t}=\lim _{t \rightarrow \infty}\left[\frac{a r^{x}}{\ln r}+\frac{d x r^{x}}{\ln r}-\frac{d r^{x}}{(\ln r)^{2}}\right]_{N}^{t} \\
= & \lim _{t \rightarrow \infty}\left[\frac{a r^{t}}{\ln r}+\frac{d t r^{t}}{\ln r}-\frac{d r^{t}}{(\ln r)^{2}}-\frac{a r^{N}}{\ln r}-\frac{d N r^{N}}{\ln r}+\frac{d r^{N}}{(\ln r)^{2}}\right] \\
= & -\frac{a r^{N}}{\ln r}-\frac{d N r^{N}}{\ln r}+\frac{d r^{N}}{(\ln r)^{2}}
\end{aligned}
$$

which is finite value for every $\mathrm{N}=1,2,3 \ldots$

$$
\therefore \int_{N}^{\infty}(a+x d) r^{x} d x=-\frac{a r^{N}}{\ln r}-\frac{d N r^{N}}{\ln r}+\frac{d r^{N}}{(\ln r)^{2}}
$$

It implies that $\int_{N}^{\infty}(a+x d) r^{x} d x$ converges and hence by integral test the given series $\sum_{k=0}^{\infty}(a+k d) r^{k}$ also converges.

Again, Upper sum $=\sum_{k=0}^{n}(a+k d) r^{k}+\int_{n}^{\infty}(a+x d) r^{x} d x$

$$
=\sum_{k=0}^{n}(a+k d) r^{k}-\frac{a r^{n}}{\ln r}-\frac{d n r^{n}}{\ln r}+\frac{d r^{n}}{(\ln r)^{2}}
$$

Similarly, Lower sum $=\sum_{k=0}^{n}(a+k d) r^{k}+\int_{n+1}^{\infty}(a+x d) r^{x} d x$

$$
=\sum_{k=0}^{n}(a+k d) r^{k}-\frac{a r^{n+1}}{\ln r}-\frac{d(n+1) r^{n+1}}{\ln r}+\frac{d r^{n+1}}{(\ln r)^{2}}
$$

Taking the special arithmetic mean of (3.3) and (3.4) we get

$$
\sum_{k=0}^{\infty}(a+k d) r^{k} \cong \sum_{k=0}^{n}(a+k d) r^{k}-\frac{a r^{\frac{2 n+1}{2}}}{\ln r}-\frac{d(2 n+1) r^{\frac{2 n+1}{2}}}{2 \ln r}+\frac{d r^{\frac{2 n+1}{2}}}{(\ln r)^{2}}
$$

assuming that mean of $\quad \int_{n}^{\infty}(a+x d) r^{x} d x \quad$ and $\quad \int_{n+1}^{\infty}(a+x d) r^{x} d x \quad$ is given by $\int_{\frac{2 n+1}{2}}^{\infty}(a+x d) r^{x} d x($ special arithmetic mean $)$

$\therefore \sum_{k=0}^{\infty}(a+k d) r^{k} \cong \sum_{k=0}^{n}(a+k d) r^{k}+\frac{r^{\frac{2 n+1}{2}}}{\ln r}\left[-a-d\left(\frac{2 n+1}{2}\right)+\frac{d}{(\ln r)^{2}}\right]$ 
This is the required result which is valid for all values of $\mathrm{a} \geq 0$ and $|r| \leq 1$

Example: 1 Estimate the sum of infinite series

$1+\frac{3}{5}+\frac{5}{5^{2}}+\frac{7}{5^{3}}+\frac{9}{5^{4}}+\frac{11}{5^{5}}+\frac{13}{5^{6}}+\cdots$

Sol $^{\mathrm{n}}$ : If $\mathrm{a}=1, \mathrm{~d}=2, \mathrm{r}=\frac{1}{5}$ and $\mathrm{n}=5$ in the equation (3.6), we get (3.7) with the sum

$$
1+\frac{3}{5}+\frac{5}{5^{2}}+\frac{7}{5^{3}}+\frac{9}{5^{4}}+\frac{11}{5^{5}}+\frac{5^{-5.5}}{\ln 5^{-1}}\left[-1-2\left(\frac{2 \times 5+1}{2}\right)+\frac{2}{\left(\ln 5^{-1}\right)^{2}}\right]=1.875097515
$$

If we apply the relation $S_{\infty}=\frac{a}{1-r}+\frac{d r}{(1-r)^{2}}$,we get the sum of (3.7) equal to $\frac{15}{8}=1.875$ which is assumed as the actual sum. We argued that as there are infinitely many terms in this series with many digits after decimals and hence it is very interesting that its infinite sum is exactly 1.875 . If we add 16 terms directly then it becomes 1.874999999725568 . Then what is the sum of remaining infinite terms? Is it equal to zero or very near to zero? So it creates some curiosity to us. In our result (3.6) if we take the different values of $n$, we get the following scenario.

\begin{tabular}{l|c|c}
\hline $\mathbf{n}$ & $\mathbf{S}_{\infty}$ & Error \\
\hline 5 & 1.875097515 & 0.000097515 \\
\hline 6 & 1.87502307 & 0.00002307 \\
\hline 7 & 1.875005327 & 0.000005327 \\
\hline 8 & 1.875001208 & 0.000001208 \\
\hline 9 & 1.87500027 & 0.00000027 \\
\hline 10 & 1.87500006 & 0.00000006 \\
\hline
\end{tabular}

Table-1 Estimated value of Infinite sum

So our finding (3.6) is quite interesting as it is being a tool that finds the more accurate value if we chose $\mathrm{n} \geq 5$ and might have some error depending upon the value of $\mathrm{n}$ which can be corrected more or less by putting the sufficient large value of $\mathrm{n}$ including some adjustment.

Now if we apply the approximation given by theorem (2.2.2) to this example, we get the following result. $S \cong \sum_{x=1}^{k} f(x)+\int_{k+1}^{\infty} f(x) d x+\frac{1}{2} f(k+1)-\frac{B_{2}}{2 !} f^{i}(k+1)-\frac{B_{4}}{4 !} f^{i i i}(k+1)-\frac{B_{6}}{6 !} f^{v}(k+1) \cdots-\frac{B_{2 m}}{(2 m) !} f^{2 m-1}(k+1)$

For $\mathrm{k}=5$ and $\mathrm{m}=3$ we will have 


$$
S \cong 1+\frac{3}{5}+\frac{5}{5^{2}}+\frac{7}{5^{3}}+\frac{9}{5^{4}}+\frac{11}{5^{5}}+\int_{k+1}^{\infty} f(x) d x+\frac{1}{2} f(k+1)-\frac{B_{2}}{2 !} f^{i}(k+1)-\frac{B_{4}}{4 !} f^{i i i}(k+1)-\frac{B_{6}}{6 !} f^{v}(k+1) \cdots-\frac{B_{2 m}}{(2 m) !} f^{2 m-1}(k+1)
$$

which gives

$$
S \cong 1+\frac{3}{5}+\frac{5}{5^{2}}+\frac{7}{5^{3}}+\frac{9}{5^{4}}+\frac{11}{5^{5}}+\frac{(2+13 \ln 5)}{\ln 5 \times 5^{6}}+\frac{13}{2 \times 5^{6}}-\frac{(2-13 \ln 5)}{12 \times 5^{6}}+\frac{(\ln 5)^{2}(6-13 \ln 5)}{720 \times 5^{6}}-\frac{(\ln 5)^{4}(10-13 \ln 5)}{720 \times 42 \times 5^{6}}
$$

\section{$=1.875345171$ (Using Online Desmos Scientific Calculator)}

Here we observed that Euler- Maclaurin formula in this modified form has less accuracy in such series.

Example 2: Estimate the sum of the series $\frac{1}{1 \times 3}+\frac{1}{5 \times 7}+\frac{1}{9 \times 11}+\frac{1}{13 \times 15}+\frac{1}{17 \times 19}+\cdots$

Soln $^{\mathrm{n}}$ : Consider a function defined by $f(x)=\frac{1}{(4 x-3)(4 x-1)}$

This function defined on $[1, \infty)$ is monotonically decreasing with positive terms and differentiable on $[1, \infty)$

Now,

$$
\begin{array}{lll}
\int_{N}^{\infty} \frac{1}{(4 x-3)(4 x-1)} d x=\lim _{t \rightarrow \infty} \int_{N}^{t} \frac{1}{(4 x-1)(4 x-3)} d x & =\frac{1}{1 \times 3}+\frac{1}{5 \times 7}+\frac{1}{9 \times 11}+\frac{1}{13 \times 15}+\frac{1}{17 \times 19}- \\
=\lim _{t \rightarrow \infty \frac{1}{8}\left[\ln \frac{4 x-3}{4 x-1}\right]_{N}^{t}} & \frac{1}{8}\left[\ln \frac{4 x-1}{4 x-3}\right]_{5.5}^{\infty} \\
=-\frac{1}{8}\left[\ln \frac{4 N-3}{4 N-1}\right] & =\frac{1}{1 \times 3}+\frac{1}{5 \times 7}+\frac{1}{9 \times 11}+\frac{1}{13 \times 15}+\frac{1}{17 \times 19}+\frac{1}{8} \times \ln \frac{21}{19} \\
& & =0.3927403847 \text { with error less than } 0.0000005
\end{array}
$$

which is finite for every N. So, by integral test the given series $\sum_{k=1}^{\infty} \frac{1}{(4 k-3)(4 k-1)}$ is convergent. We now estimate the sum by using our special mean method as

$$
\begin{aligned}
& \sum_{k=1}^{\infty} \frac{1}{(4 k-3)(4 k-1)} \\
& \cong \sum_{k=1}^{n} \frac{1}{(4 k-3)(4 k-1)}+\int_{\frac{2 n+1}{2}}^{\infty} \frac{1}{(4 x-3)(4 x-1)} d x
\end{aligned}
$$

If we take $n=10$ then this sum becomes 0.3927042784 while $\mathrm{n}=20$ gives 0.39269973240 which is accurate to 6 decimal places where the error is decreasing depending on the value of $\mathrm{n}$ but if we add directly then the sum of 500 terms only gives 0.3925740817299737 which has more error than our result by

taking only 5 terms. Its accurate value is taken as $\frac{\pi}{8}$ [Barbour, 1881]. So, in this case also our approximation is more appropriate. Thus, we generalize this concept in the form of following theorem.

When $\mathrm{n}=5$ then the relation gives the value as

$$
\begin{aligned}
& \sum_{k=1}^{\infty} \frac{1}{(4 k-3)(4 k-1)} \\
& \cong \sum_{k=1}^{5} \frac{1}{(4 k-3)(4 k-1)}+\int_{5.5}^{\infty} \frac{1}{(4 x-3)(4 x-1)} d x
\end{aligned}
$$

Theorem 3.2 (Generalization): Let $f(x)$ is a continuous, positive and decreasing function of $x$ defined on $[0, \infty)$. Then the infinite sum of series formed by this function can be approximated by the relation $S \cong \sum_{x=1}^{k} f(x)+\int_{\frac{2 k+1}{2}}^{\infty} f(x) d x$ 


\section{CONCLUSION}

In this research study, we reviewed a small part of Euler's work in infinite series containing the positive terms which are monotonically decreasing. By following his work, we established that the infinite series of type

$$
\sum_{k=0}^{\infty}(a+k d) r^{k} \text { where }-1<\mathrm{r}<1 ; \mathrm{a} \geq 0
$$

converges approximately to

$$
\sum_{k=0}^{n}(a+k d) r^{k}+\frac{r^{\frac{2 n+1}{2}}}{\ln r}\left[-a-\frac{d(2 n+1)}{2}+\frac{d}{\ln r}\right]
$$

for $\mathrm{n} \geq 5$. By illustration of some examples and using this concept we also established a theorem given by "For any function $\mathrm{f}(\mathrm{x})$ which is a continuous, positive and decreasing and defined on $[0, \infty)$ such that its infinite sum formed by this function can be approximated by the relation $S \cong \sum_{x=1}^{k} f(x)+\int_{\frac{2 k+1}{2}}^{\infty} f(x) d x "$

In these days many problems in applied mathematics have been solved in the form of infinite series and hence it needs very easy method of approximation of the sum. In this sense our method also plays an important role. Moreover, other researchers can extend such methods of approximation that may help in computer programming too.

\section{REFERENCES}

Barbour, L.G., Infinite series, The Analyst, V.18, n.6, p.191192, 1881

David, Benko, The Basel problem as a telescoping series, College math journal, v.43, n.3, p.244-250, 2012.

Dunham W., Euler: The Master of Us All, Dolcian Mathematical expositions, pp 22,1999.

Ehrenburg, D. O., Shortcut to Summation of Infinite Series. Mathematics Magazine, v. 39, n. 2, p. 93-96,1966.

JSTOR, www.jstor.org/stable/2688720. Accessed 5 July 2020.

Erdos Paul and Dudley Underwood, Some Remarks and Problems in number theory related to the work of Euler. Mathematics Magazine, v.56, p.292-298, 1983.

Gautschi Walter, Leonhard: His Life, The Man, and His Works, SIAM Review, v.5, n.1, p.3-33, 2008.

Knopp, K. Theory and Application of Infinite series, New York: Dover, p.117-160, 1990.

Linda Becerra and Elias Y. Deeba, Euler and Bernoulli numbers and Series Representation of Some Elementary Functions, Pi-Mu Epsilon Journal, v.12, n.9, p.521-528, 2008.

Mishra,V.N., Some Problems on Approximations of Functions in Banach Space, Ph.D. Thesis, Indian Institute of Science and Technology, Roorkee247667, Uttarakhand, India,2007
Mishra, V.N.and Mishra L.N., Trigonometric Approximations of Signals (Functions) in $\mathrm{L}_{\mathrm{n}}$-norm, International Journal of Contemporary Mathematical Sciences, v.7, n.19, p.909-918, 2012.

Moris Kline, Euler and Infinite series, Mathematics Magazine. v.56, n.5, p.307-314, 1983.

Nelson, Roger B., An Improved Remainder Estimate for use with the Integral Test, The College Mathematical Journal, v.34, n.5, p.397-399, 2003.

Nick Lord, Beyond the Bassel Problem: Euler's Derivation of the general formula $\xi(2 n)$, The Mathematical Magazine, vol.98(543). Pp .459-474, 2014.

Paudyal, D.R., A Short Note On Basel Problem, BMC Journal of Physics, v.2, n.1, p.38-46, 2014.

Pfaff,T.J., Deriving a formula for sums of power of integers, The PME Journal, V.12, p .425-430, 2007

Raymond J.Collins, Approximation Series, The College Mathematical Journal, v.23, n.2, p.153-157,1992.

Stanattis, O.E., On Approximation of slowly convergent series, Time Journal, v.13, n.1, p.186-190, 2009. 